Marquette University

e-Publications@Marquette

College of Nursing Faculty Research and

Publications

Nursing, College of

6-1-2012

\title{
Clinical Observation Reflections from Students in an Interdisciplinary Palliative Care Course
}

Marcia Phillips

Susan Breakwell

Marquette University

Kim MinJu

Margaret Faut Callahan

Marquette University

Author version. Journal of Hospice and Palliative Nursing, Vol. 14, No. 4 (June 2012): 274-282. DOI.

(C) 2012 The Hospice and Palliative Nurses Association. Used with permission. 


\title{
Clinical Observation Reflections from Students in an Interdisciplinary Palliative Care Course
}

\begin{abstract}
The purpose of this study was to gain insight into how a brief clinical observation encounter contributed to students' experiences in an interdisciplinary palliative care course. This course was required of all graduate nursing students and was available as an elective for medical and other healthcare professions students at a healthcare sciences university. The students were required to spend approximately 8-12 hours attending interdisciplinary team meetings or accompanying a team on rounds and patient visits.

The students' summary narratives of their observation experience were analyzed in this qualitative study that focused on six categories of feedback: 1) patients' and families' reactions, 2) communication issues with patients and families, 3) how the palliative care team speaks with the patient and family, 4) communication within the interdisciplinary team, 5) students' reflections, and 6) students' suffering.

This study demonstrated that a clinical observation activity can be a valuable introduction to palliative care principles for healthcare students in an interdisciplinary course. Students benefited from gaining insight into family/practitioner communications regarding difficult issues, interdisciplinary roles and cooperation, and application of palliative care principles to clinical practice. Further research is required to identify appropriate interventions to deal with student distress resulting from such early career clinical encounters.
\end{abstract}




\section{Introduction}

\section{Description of Clinical Observation in Palliative Care}

In 2006, an interdisciplinary palliative care course required of all graduate nursing students and optional for medical and other allied health care students was initiated at a large academic medical center. This course focuses on developing students' knowledge and application of palliative care and interdisciplinary team concepts by engagement in online content and discussions, simulation laboratory exercises, and clinical observation (see Table 1). The course is a graduate level two-credit course offered multiple times throughout the year. One of the course activities is a clinical observation of palliative care services where students spend approximately $8-12$ hours attending an interdisciplinary team meeting (IDT), and/or accompanying a palliative care team on rounds and/or accompanying a team member on patient visits to inpatient and/or outpatient settings. The objectives of this course activity are to: (1) describe how interdisciplinary palliative care team principles can be applied in a clinical setting, (2) identify characteristics of a palliative approach in the care of patients, and (3) identify the team members' role in incorporating a palliative approach to care. Afterwards, each student composed a brief summary narrative of their observation.

"Interdisciplinary" is a term used in three different contexts. Interdisciplinary is used when referring to palliative care teams as defined by the National Consensus Project for Quality Palliative Care as "a core group of professionals from medicine, nursing and social work, and may include some combination of volunteer coordinators, bereavement coordinators, chaplains, psychologists, pharmacists, nursing assistants and home attendants, dietitians, speech and language pathologists, physical, occupational, art, play, music, and child-life therapists, case

managers, and trained volunteers." ${ }^{1}$ It also refers to an interdisciplinary palliative care course 
that includes degree seeking students from different disciplines (nursing, medicine, and other allied health care programs). Finally, the faculty participating in this course is referred to as interdisciplinary and consists of nurses, physicians, chaplains and other allied health care professionals.

\section{Table 1.}

Course Activities, Methods and Content

\section{Purpose}

The purpose of this study was to gain insight into how a brief clinical observation activity contributed to students' experiences in an interdisciplinary palliative care course. Two key questions were asked: Is a brief clinical observation activity meaningful to students in an interdisciplinary palliative care course, and what do students self-identify as learning that occurred? To explore these questions students' summary narratives of their observations were studied.

\section{Methods}

A university Institutional Review Board approval was obtained. At the beginning of the course, each student received a description of the assignment, including the objectives, anticipated time, and a list of participating palliative and hospice care sites. Students then selected a site and arranged for their clinical observation. At the selected site, students accompanied a team or team member engaged in palliative care work. After their observation, the students were to prepare a brief summary narrative of the site and activities, including any 'lessons learned' and post in a designated discussion board section within the online course shell. 
These broad, non-directed instructions were designed to allow students flexibility in addressing aspects of the observation that were of greatest significance to them. These summary narratives were gathered for the period of January - August 2010. The study time period coincided with the initiation of a new online teaching system. At that time the course was well established with earlier revisions already incorporated, ensuring to the degree possible that faculty, course activities, and expectations of students remained consistent over time.

\section{Participants}

The summary narratives for this study originated from 207 nursing, medicine and other allied health care students taking this course in 2010. There was a diversity of participants, the sample size was adequate for qualitative data collection, and the clinical settings were experienced in working with students completing the summary narrative of their observation activity associated with this course.

Students were predominately female $(81.2 \%)$ and enrolled in post-licensure advanced practice nursing $(n=106)$, pre-licensure generalist entry master's $(G E M)$ program in nursing $(n=$ 55), medical students in their clerkship $(n=42)$, or other health care programs $(n=4)$. Students' palliative care observations occurred in a variety of settings: hospitals $(n=141)$, hospices $(n=$ 64), and other settings (a nursing home and a retirement residence) $(n=2)$.

\section{Data analysis}

The data were organized and managed using QSR NVivo 8 (QSR International, Melbourne, Australia). Identifying information was removed and an alpha numeric code was assigned to each summary. The data were analyzed using the approach developed by Ritchie and Spencer $^{2}$ (1994). They were sifted, charted, and sorted in accordance with emerging key issues and themes through a five step process of analysis: familiarization with the context, identifying a 
thematic framework, indexing themes, charting themes, and mapping and interpretation of themes. Three authors reviewed the summary narratives to gain an understanding of the collected data and to identify key ideas and recurrent themes. This stage of the review originally revealed 10 themes including students' reflection and suffering; reactions of family and patients; issues and problems in communication with patient, family, and other health care providers; and how to approach discussion of difficult issues. In subsequent discussion among the authors, a thematic framework was developed, organizing identified themes into categories. The resulting reconceptualization revealed 6 categories: 1) patients' and families' reactions; 2) communication issues with patients and families; 3) how the palliative care team speaks with the patient and family; 4) communication within the interdisciplinary team; 5) students' reflections; and 6) students' suffering (see Table 2). After applying these six categories and the associated themes in combination with the overall study aims, each of the summary reports were indexed and charted. One of the reviewers then re-coded the summaries, organizing them in the revised categories. Finally, three authors reviewed the newly reconfigured categories, recoding, mapping, and interpreting themes that emerged in the summary narratives. Through further communication, any discrepancies were clarified and resolved by consensus. ${ }^{3}$

Table 2.

Categories and Corresponding Themes Emerging From a Palliative Care Observation

\section{Results}

The summary narratives varied widely; some students reported 'just the facts' while others described how the principles covered in the course materials were applicable to the 
observation experience. Remaining descriptions were at a synthesis level, linking what had been learned in the course with what was (or was not) seen, and ascribing potential recommendations and meaning for future practice at a personal, discipline, or systems level. The remainder of this section describes the findings for each of the six categories shown in Table 2.

\section{Patients' and families' reactions}

One category identified in student summary narratives $(n=30)$ was the difficulty families and patients had in accepting bad news about their health condition and/or recommendations. The narratives described families' reactions as 'very upset', 'crying', 'a difficult time talking about the subject', 'hesitant to talk', 'very emotional', 'saddened', 'continued to ask for other options', 'abruptly left the room', 'somewhat combative', 'distraught', 'extremely overwhelmed', and so forth. Many students also described patients' negative reactions when they heard the options of hospice and palliative care as 'completely resistant to any conversation about palliative care or hospice care' and 'resistant to discuss goals of patient care'.

Within this category, the theme of misunderstanding about hospice and palliative care was one identified barrier for patients and families, as noted by the comment, "Both he [the patient] and his wife believed hospice care meant sending the patient home, drugging him up until he died." Hospice and palliative care were often understood as 'giving up' by families and patients described as "abandoning him [the patient]."

Both barriers and opportunities for families making end-of-life decisions were identified as another theme. There was recognition of difficulties, such as 'feeling guilty' and 'honoring the patient's wishes and advance directives', as in the example, "She [a daughter] wanted to do everything possible to save her [the patient] and said that she was the one that would have to live with herself if her mother died without resuscitating her." 
The importance of advance care planning was noted. As described by a case in which the family members had known the patient's wishes for end-of-life care, “...the husband knew through discussions with his wife that she did not want anything invasive done to save her life..... All of the family was in agreement that comfort care would be the right step ....."

\section{Communication Issues with patients and families}

When reflecting on issues in communication with patients and families, students $(n=38)$ identified several issues, such as differences in goals of care among patients, families, and health care professionals, the importance of the use of interpreters, unclear patients' wishes, and lack of family involvement.

In many of the summary narratives, students identified differences between patient/family and health care professionals as the main communication issue, attributing it to lack of communication with the primary health professionals and reluctance to discuss end-oflife care. Highlighting communication concerns, narratives noted, "She was not sure exactly what her other options were and was frustrated with the communication she was not receiving from her oncologist" and "It was a shock to the family because they had no idea the patient's prognosis was so poor at this time." As an example of reluctance to discuss end-of-life care, a student noted, "the family decided to go with ... [the patient's] wishes to go home, but not to tell him that he was dying."

In addition, unrealistic hope and misunderstanding about palliative care, hospice care, and pain management medication were associated with a difference in goals of care between the patient/family and health care professionals. The importance of the use of an interpreter in communicating with patients and families who could not speak English, as well as communication challenges when they were absent, was recognized in many of the summary 
narratives. Since the interpreter could not always be with the patient and family, it was hard for the patient and family to get information and express their concerns to health care professionals. Students noted: "Due to communication difficulties, the care for their mother up to the current time had not been what they had wanted" and "The only family member that spoke English was the granddaughter who was probably between the age of 17-19 and seemed a bit overwhelmed with translating some of the things that the team had to say to the family."

Students identified the importance of knowing the patient's wishes. In one example, "The patient.....didn't have his wishes in writing; his partner was the only one who explained what the patient's wishes were. However, the mother of the client had different ideas about how her son has to be buried and what services had to be provided." In addition, a lack of family involvement is another issue communicated. A student noted, “...the wife was the surrogate decision maker however, she never seemed to visit the patient often and seemed recalcitrant to the calls by the interdisciplinary team to sit down and have a meeting about further plans for care."

\section{How the palliative care team speaks with the patient and family}

Of all the communication-focused categories in this study, how the palliative care team spoke with patients and families was most frequently addressed in students' summary narratives $(n=74)$. They were impressed by verbal and non-verbal communication skills used in working with patients and their families. Additionally, they recognized the value of these skills in developing goals of care. Palliative care team approaches to these communications were characterized as "listening to", "non-threatening”, "gentle", "empathy”, “clear information”, "using easy terms", "slowly", "willing to spend the time", "honest", "compassionate", "respectful", "mutual respect", and "comforted." As reflected in one narrative, "The empathetic listening, calm and informative advice and thorough medical knowledge resonated with me", 
while another found, "It is an art to lead these discussions and guide the patient allowing them time to come to their own conclusion without directly stating your opinion."

Students noted how effective non- verbal techniques contributed to communication with families. For example, “... [the physician] sat next to them or even on their bed. His voice was soothing and he often touched their hands as he spoke." and "They held patients hands, crouched down for leveled eye contact, and listened with caring demeanor."

Flexibility was recognized as an important attribute for a palliative care team faced with sensitive or difficult communications. As characterized in one summary, "The team suggested a creative plan...Both scenarios showed a flexibility of the teams to get the patient home... [The team] was quite careful not to take away the mother's hope or attempt to diminish her faith, but... was also honest in the prognosis and probable course of illness. ...It was inspiring to see her demeanor and the relationship that she and the mother were able to maintain out of that mutual respect."

\section{Communication within the interdisciplinary team}

Themes identified by students $(n=31)$ in the category of "communication within the interdisciplinary team' were about positive and effective communications; some ineffective communications were also noted. From observations of interdisciplinary team meetings, it was recognized that enhancing comfort and quality of life of patients and their families, as well as the unique roles of team members, were central to interdisciplinary team meetings. As one student noted, "At the end of the day, all members did have the patient's best interest as their priority and it was interesting to see them collaborate on the plan of care." Repeatedly, the unique contributions of individual team members and the effectiveness of communication among team members were addressed in narratives. For example, "All of the disciplines brought something 
unique to the plan of care and really communicated well and worked together to problem solve any issues that arose."

Several students recognized ways that an individual's communication skills contributed to the interdisciplinary team itself, describing them as "very aware of his or her role in the care of the patient", "voiced his or her concerns for improving the patient's quality of life", "definitely engaged and always brought forward a useful perspective", and "very informative and in depth ."

Students were inspired by activities, rituals, or ceremonies that team members at hospices engaged in as remembrance of those who died the previous week. The interdisciplinary team placed "a representative stone into a jar for each patient who passed", "lit a candle", and added "a marble to a bowl of marbles for each passing." During this ceremony, the team members had an opportunity to share their experiences with the patients and their families. Sometimes a journal was sent to each of their families. Students identified these activities as support not only for the family in bereavement but also for the health care professionals.

Negative aspects of communication observed within an interdisciplinary team included tension among the team and a lack of resources. In addition, a student's experience showed the lasting impression an observed encounter of palliative care and primary health care professionals can have. One summary noted: "The doctor and nurse were condescending to us and acted as though we were invading their space. We left with nothing accomplished because the doctor refused to discharge the patient..."

\section{Students' reflections}

Almost one half of the students $(n=100)$ reflected on the work of the palliative care team in their summary narratives. Among their observations was a recognition of the importance of 
better understanding of the views of the patient and the family and of tailoring communication to the needs and situation at hand. As described in one summary narrative, "I had to put myself in the family members' shoes... I think sometimes the details can be an important focus for family members, who might feel confused or hopeless otherwise...it gives them something to focus on or short term goals to achieve, which can provide hope, no matter the actual utility of those goals."

In the palliative care literature, interdisciplinary teams were defined as: "...palliative care teams, with members contributing from their particular expertise. The team shares information and works interdependently." ${ }^{4}$ Students' understanding of interdisciplinary palliative care teams working in collaboration is a key principle that emerged through their narratives. Comments recognizing the principles of patient and family centeredness and goals of care, included, "what is best for the patient and treat her to the best of our ability" and "the patient as the center, rather than the 'medicine' at the center." The importance of interdisciplinary collaboration was apparent, as exemplified in one narrative, "a collaborative practice between nurses, doctors, chaplains and other health care providers."

Emerging from summary narratives were observations of how the palliative care team members worked with each other and communicated with the dying patients and their families. Students reflected on how observing these encounters would potentially impact their future practice. They also were attuned to similarities and contrasts in practice between this observation and other clinical experiences. As described by one student, "It was a drastic contrast from what we are accustomed to seeing on the medical floors during our regular clinical hours, and this experience has provided insight about patient interaction and therapeutic communication that will carry over into my practice regardless of specialty." This experience was helpful to address 
another's fear about death and dying, noting that "The hospice nurse... helped alleviate any fears or preconceived notions I may have had or imagined about the dying process."

The need for support of the team members was recognized in the summary narratives. Consistent with this theme were such reflections as, "It was definitely something to take into consideration because the EOL process can be time consuming and emotionally stressful. This on top of your own personal life can take a toll on an individual" and "I was struck by the emotional burden that the nurses must carry as they get to know patients and families and then experience the deaths of these patients that they come to know."

\section{Students' suffering}

For the purposes of this study, student suffering was extrapolated from the students' summary narratives, in which the students expressed their personal emotions of distress and despair in describing their observation experiences. Summary narratives described a range of emotional responses to the observation experiences. Some students $(n=9)$ ascribed aspects of suffering to their observations, particularly with dying patients and their families, or when there were conversations about difficult news, decisions, and goals of care. "I and another student were in the corner trying to hold back tears. It was a very tough but touching moment"; "I will never forget the look of despair in her [patient] eyes as she [patient] said, 'so this is it'. It was definitely heartbreaking for me to watch someone get that news." "That was difficult to watch... This particular situation was very surreal for me. It was very difficult to watch the family dynamic as they received this horrible news" was the reaction of one student. Similarly, in another summary, "I have never been in a room with a patient's family like that before. They moved me to tears."

\section{Discussion}


Six key categories were identified through the summary narratives describing time students spent observing palliative care teams and individual members as part of their palliative care course. Students gained valuable insights in six categories: patients' and families' reaction; communication issues with patients and families; how the palliative care team speaks with the patient and family; communication within the interdisciplinary team; student's reflection; and student's suffering. For many this observation time was their first opportunity to be present with patients nearing the end of life and their families. Students' observations were often in a one day period so their opportunities to see health care team members, patients, and families over time were limited. This hampered their ability to observe communication techniques, palliative measures, and the evolution of coping strategies over time. The structure of the experience did, however, allow students to be exposed to varied patients and families, diagnoses, prognoses, and the work of interdisciplinary palliative care teams.

In the patients' and families' reactions category, many comments addressed misunderstandings about the terminology of palliative care and hospice, which is not uncommon. ${ }^{5,6}$ The summary narratives identified how difficult it was for patients and families to accept bad news. A range of reactions to difficult news were described. Patient and family denial and anger can be manifested in many ways, and their reactions of overwhelming distress and emotion were recognized by students.

The second category, observing communication issues with patients and families, added to the students' learning experience. Students identified how the teams worked through confusion or misunderstandings with patients and their families, and observed important strategies that enhanced or created barriers to communication. For patients and families, clarification about care options from healthcare providers seemed to decrease their level of 
frustration in making decisions. Students observed how lack of communication with primary health care providers and reluctance to discuss end-of-life issues hampered communications. The students recognized the necessity of language interpreters as part of culturally respectful care provision. Their observations parallel literature about facilitating interpretation between the health care professionals, patients, and their families. ${ }^{7,8}$ Observing what worked well and what did not work so well could provide the students new ideas about ways of dealing with similar situations in their future positions.

Captured in the third category, students were impressed by how the palliative care team speaks with the patient and family. The teams' empathy and compassion when speaking and listening to the patients and their families as they told their stories was recognized in the summary narratives. Students were often impressed by how the team started difficult conversations. When delivering bad news to patients and their families, students recognized that the team members' effective communication strategies were similar to those from their readings about the "SPIKES" tool for delivering difficult news. ${ }^{9}$ Elements of the tool noted most by students were: eliciting the patient's perception of their condition ("P"), inviting conversation ("I"), and empathizing ("E"). Hearing the health care teams' specific words and viewing their actions during these observation moments can leave an indelible impression regarding sensitive communication and provide new strategies for dealing with difficult conversations in future practice. The literature discusses the importance of communication between cancer patients and palliative care professionals in helping provide better care to those patients. ${ }^{5,10,11}$

The fourth category from the study was communication within the interdisciplinary team itself. Students noted a range of communication effectiveness within the teams. Most worked well together, supported each other and ensured that each member's voice was acknowledged. 
Some teams' communications were more strained and not as supportive. Raised frustration and tension levels led to team disillusion, ineffective communication, and inaction, and distanced the team's focus from the patient and family. Valuable learning experiences can occur when students observe both positive and negative communications and their consequences. ${ }^{10}$ Students observed the unique contributions of each team member and how these roles contributed to the development of the patient's plan of care.

Several themes emerged from the category of students' reflections. One was the importance of understanding the wishes of the patients and families as they attempted to imagine themselves in similar situations and how they might react. Their reflections were verbalized as "now understanding", "I felt", "I thought" and "I am truly convinced" with comments related to patient-centered care and collaborative practice. Many reflected that observing the teams' communication would have a positive impact on their future practice. One comment of note was that during one's observation, any fears imagined about the dying process were alleviated. Lastly, several students reflected on the emotional burden the staff and palliative care team carried and the extensive amount of time it takes caring for patients with advanced illness or at the end stage of life and their families.

The last category, about issues surrounding students'suffering, emerged somewhat unexpectedly. Some students expressed feelings of how difficult it was for them to observe an encounter when the team delivered bad news. They mentioned being brought close to tears while viewing heartbreaking cases, encapsulated by the statement "I will never forget the look of despair in her [patient] eyes as she [patient] said, 'so this is it'. " This comment and others with phrases such as: "It was heart wrenching to hear..."; "It was difficult to watch..."; and "I have never been in a room with a patient's family like that before. They moved me to tears." led to a 
realization that actual or potential student suffering might be occurring, despite the brief nature of the observation. For many, this was their first end of life encounter with patients, their loved ones and the interdisciplinary team. It was recognized that this could be an experience of impact for the student beyond the end of the course and associated observation.

Results from our analysis of students' summary narratives suggest that these learning experiences affected them personally and professionally. As others have observed from similar studies, students were aware of the value of hospice and palliative care, identified the role of palliative care team members and understood death as a natural event through learning experiences in palliative care and hospice settings. ${ }^{12,1314}$ Unlike other studies with students in one discipline ${ }^{12-15}$, this study could be useful in understanding the dynamics of incorporating teamwork concepts within an interdisciplinary educational experience. In addition, other studies did not consider student suffering that could result from this type of clinical observation experience.

\section{Implications}

With the majority of the communication being online for this course, written summary narratives of students' observation experiences were a valuable tool. They provided students the opportunity to reflect and to ascribe meaning to their observations. It also provided a vehicle for synthesizing what was learned in the course with what was seen in a practice environment, furthering the aim of integration of palliative care principles into future practice.

One question that came to mind upon reviewing the summary narratives data was: Were there instances of despair or suffering that students did not address in their write-ups? While the course was designed so that course faculty active in facilitating the discussion board could respond to a student's summary narrative within the discussion board itself, and/or on a private 
and individual basis via course mail, other mechanisms for identifying students working through questions or challenges associated with the observation can be developed. To do so, faculty need to continue to recognize that some students may be reluctant to initiate or are uncomfortable in discussing feelings online. Additionally and unknown to faculty, other personal, home, or school issues may contribute to students' reactions during their observation experience.

Course directors can facilitate students' processing of distress or grief experienced during the course in various ways. Providing information early on in the course syllabus and introductory online discussions, as well as recognizing that the course contents can be sensitive in nature can help set an open, welcoming tone for students. In addition, addressing expectations for using proper online etiquette can help guide students in developing appropriate techniques for posing questions, comments, or concerns. Coordinating the observation experiences with clinicians and sites/services known to the faculty ensured mutual understanding about expectations of everyone involved. By monitoring the discussion boards, and other individual/private communications from students, faculty was able to respond to students' questions or issues during the course.

Additional faculty efforts to reach out to students can include an introductory note from faculty recognizing that that it is not uncommon for students to encounter a range of feelings during this course, especially during their clinical observation experience, and guiding or inviting them to discuss these issues among course participants and/or solely with the course faculty. Faculty need to be available to listen, offer guidance, and/or provide additional resource information, such as counselors, to students actively seeking support. Some students who are not proactive or comfortable in asking for support may benefit from faculty reaching out to them, thus underscoring the value of faculty vigilance in thoroughly understanding students' summary 
narratives. In this way, faculty can determine whether to acknowledge observation experiences that were difficult in a group discussion, and/or follow up privately with the individual via email, phone, or in person.

The students' work in this course prior to their observation experience consisted of online readings and discussions regarding palliative and end of life care. The real situations encountered in the actual observation experience helped students apply and synthesize course concepts with practice and to reflect privately and/or among their course colleagues in online postings.

\section{Conclusion}

This study demonstrated that a clinical observation activity can be a valuable introduction to palliative care principles for healthcare students in an interdisciplinary course. Though brief in terms of time, students identified a number of issues related to communication, interdisciplinary teams, patients and families, and personal reflections. Students were exposed to the benefits of a team approach to palliative care, the importance of appropriate communication strategies between healthcare professionals and their patients and patients' families, and communications among the team members themselves. Through their summary narratives, students were able to link information from their coursework to situations they saw and heard in practice, reinforcing that learning had occurred.

An interesting issue uncovered by this study is the need to have a greater awareness that students may experience a range of reactions. Some students reflected on feelings of distress, sadness, or suffering in their summary narratives, attributed at least in part to never having been present with patients who were so near the end of life. This raised faculty's awareness and reinforced the continued need to encourage students to contact faculty for follow up assistance if they experience any difficulty dealing with emotions or would like to talk about their reactions 
related to the observation experience or other aspects of the course. Recognition of the range of potential student reactions to end of life issues remains an important consideration in future curricular development.

The clinical observation experience appears to be a valuable part of an interdisciplinary palliative care course. Important palliative care issues, including patients' and families' reactions to difficult news and communications with patients and palliative care teams, emerged clearly in students' summary narratives, illustrating the impact of this observation experience on students' understanding of palliative care issues. Observation experiences require a commitment in resources beyond usual didactic instruction. This study has reinforced the value of such exposures to developing awareness and skills. 


\section{References}

1. National Consensus Projects for Quality Palliative Care. Clinical practice guidelines for quality palliative care, Second Edition. 2009.

http://www.nationalconsensusproject.org/guideline.pdf. Accessed December 26, 2011.

2. Ritchie J, Spencer L. Qualitative Data Analysis For Applied Policy Research London \& New York: Routledge; 1994.

3. Creswell JW. Qualitative Inquiry and Research Design. London: Sage Publication; 1997.

4. Crawford GB, Price SD. Team working: palliative care as a model of interdisciplinary practice. Med J Aust. Sep 15 2003; 179(6 Suppl): S32-34.

5. Batchelor NH. Palliative or hospice care? Understanding the similarities and differences. Rehabil Nurs. 2010; 35(2): 60-64.

6. Bonebrake D, Culver C, Call K, Ward-Smith P. Clinically differentiating palliative care and hospice. Clin J Oncol Nurs. 2010; 14(3): 273-275.

7. Kemp C. Cultural issues in palliative care. Semin Oncol Nurs. 2005; 21(1): 44-52.

8. Norris WM, Wenrich MD, Nielsen EL, Treece PD, Jackson JC, Curtis JR.

Communication about end-of-life care between language-discordant patients and clinicians: insights from medical interpreters. J Palliat Med. 2005; 8(5): 1016-1024.

9. Baile WF, Buckman R, Lenzi R, Glober G, Beale EA, Kudelka AP. SPIKES-A six-step protocol for delivering bad news: application to the patient with cancer. Oncologist. 2000; 5(4): 302-311.

10. Fallowfield L, Jenkins V. Effective communication skills are the key to good cancer care. Eur J Cancer. 1999; 35(11): 1592-1597.

11. Hjorleifsdottir E, Carter DE. Communicating with terminally ill cancer patients and their families. Nurse Educ Today. 2000; 20(8): 646-653. 
12. Maxwell TL, Passow ES, Plumb J, Sifri RD. Experience with hospice: reflections from third-year medical students. J Palliat Med. Oct 2002; 5(5): 721-727.

13. Jacoby LH, Beehler CJ, Balint JA. The impact of a clinical rotation in hospice: medical students' perspectives. J Palliat Med. Jan 2011; 14(1): 59-64.

14. Kwekkeboom KL, Vahl C, Eland J. Companionship and Education: A Nursing Student Experience in Palliative Care. J Nurs Educ. 2005; 44(4): 169-176.

15. Wittenberg-Lyles EM, Goldsmith J, Ragan SL, Sanchez-Reilly S. Medical students' views and ideas about palliative care communication training. Am J Hosp Palliat Care. Feb 2010; 27(1): 38-49. 
Table 1

Course Activities, Methods and Content

\section{Pre and post course}

Students complete online self assessments of knowledge and perceived effectiveness

\section{Content}

Students complete web-based didactic modules and participate in faculty facilitated asynchronous online small group discussions

Module 1: Palliative Care and Interdisciplinary Teams

Module 2: Pain and Pain Management

Module 3: Symptoms and Symptom Management

Module 4: Suffering and Reflective Practices

Module 5: Decision Making

Module 6: Care in the Final Hours

\section{Simulation laboratory}

Students participate in a 3 hour in-class or web-based, case- centered faculty facilitated role play, group discussion, and complete a post session self-reflection

Case 1: Patient with lung cancer over time (from pre- diagnosis to advanced disease)

Case 2: Patient with advanced dementia and family decisions about advance directives and care

Case 3: Patient with late stage cancer and concerns about the family

\section{Clinical observation}

Students observe clinicians and care at palliative care service rounds, interdisciplinary team meetings, and/or hospice visits for $1-1 \frac{1}{2}$ days, then summarize their observation in an online discussion board facilitated by course faculty.

Interdisciplinary palliative care and hospice team principles

Characteristics of palliative and hospice approaches to care

Health care team members' roles in palliative and hospice care provision 
Table 2

Categories and Corresponding Themes Emerging From a Palliative Care Observation

\begin{tabular}{l} 
Category \\
\hline $\begin{array}{l}\text { Patients' and families' reactions } \\
(\mathrm{n}=30)\end{array}$ \\
Communication issues with patients
\end{tabular}
and families $(n=38)$

\section{Themes}

- families' reactions

- misunderstanding about hospice and palliative care

- barriers and opportunities for families making end of life decisions

- importance of advance care planning

- differences between patient/family and health care professionals

- misunderstanding about palliative care, hospice, and pain management medication

- use of an interpreter

- knowing the patient's wishes

How the palliative care team speaks with the patient and family $(n=74)$

Communication within the interdisciplinary team $(n=31)$

Students' reflections $(\mathrm{n}=100)$

Students' suffering $(\mathrm{n}=9)$

- verbal and non-verbal communication skills

- skills in developing goals of care

- team skills surrounding sensitive/difficult goals of care communications

- unique roles of team members

- effectiveness of communication among team members

- team rituals/activities/ceremonies

- negative communications

- understanding of the views of the patient and the family

- tailoring communication to needs/situations

- importance of collaboration

- team work and communicating with dying patients and families.

- potential impact on future practice

- similarities/contrasts between observation and other practice/experiences.

- need for support of the team members

- personal feelings of sadness

- difficult observation/experience

- tears of emotion 